\begin{tabular}{|c|l|}
\hline Title & $\begin{array}{l}\text { Longitudinal and transverse exciton- spin relaxation in a single InA sP quantum dot embedded inside a standing InP } \\
\text { nanowire using photoluminescence spectroscopy }\end{array}$ \\
\hline Author(s) & $\begin{array}{l}\text { Sasakura, H.; Hermannstädter, C.; Dorenbos, S. N.; A kopian, N.; van Kouwen, M. P.; Motohisa, J.; Kobay ashi, Y.; } \\
\text { Kumano, H.; Kondo, K.; Tomioka, K.; Fukui, T.; Suemune, I.; Zwiller, V. }\end{array}$ \\
\hline Citation & $\begin{array}{l}\text { Physical Review B, 85(7), 075324 } \\
\text { https://doi.org/10.1103PhysRevB.85.075324 }\end{array}$ \\
\hline Issue Date & 2012-02-15 \\
\hline Doc URL & http://hdl.handle.net/2115/48661 \\
\hline Rights & O2012A A merican Physical Society \\
\hline Type & article \\
\hline File Information & PRB85-7_075324.pdf \\
\hline
\end{tabular}

Instructions for use 


\title{
Longitudinal and transverse exciton-spin relaxation in a single InAsP quantum dot embedded inside a standing InP nanowire using photoluminescence spectroscopy
}

\author{
H. Sasakura, ${ }^{1, *}$ C. Hermannstädter, ${ }^{1}$ S. N. Dorenbos, ${ }^{2}$ N. Akopian, ${ }^{2}$ M. P. van Kouwen, ${ }^{2}$ J. Motohisa, ${ }^{3}$ Y. Kobayashi, ${ }^{3}$ \\ H. Kumano, ${ }^{1}$ K. Kondo, ${ }^{1}$ K. Tomioka, ${ }^{3,4}$ T. Fukui, ${ }^{3,4}$ I. Suemune, ${ }^{1}$ and V. Zwiller ${ }^{2}$ \\ ${ }^{1}$ Research Institute for Electronic Science, Hokkaido University, Sapporo 001-0021, Japan \\ ${ }^{2}$ Kavli Institute of Nanoscience, Delft University of Technology, NL-2628 CJ Delft, The Netherlands \\ ${ }^{3}$ Graduate School of Information Science Technology, Hokkaido University, Sapporo 060-0814, Japan \\ ${ }^{4}$ Research Center for Integrated Quantum Electronics (RCIQE), Hokkaido University, Sapporo 060-8628, Japan
}

(Received 15 February 2012; published 29 February 2012)

\begin{abstract}
We have investigated the optical properties of a single InAsP quantum dot embedded in a standing InP nanowire. Elongation of the transverse exciton-spin relaxation time of the exciton state with decreasing excitation power was observed by first-order photon correlation measurements. This behavior is well explained by the motional narrowing mechanism induced by Gaussian fluctuations of environmental charges in the nanowire. The longitudinal exciton-spin relaxation time is evaluated by the degree of the random polarization of emission originating from exciton states confined in a single-nanowire quantum dot by using Mueller calculus based on Stokes parameters representation. The reduction in the random polarization component with decreasing excitation power is caused by suppression of the exchange interaction of electron and hole due to an optically induced internal electric field by the dipoles at the wurtzite and zinc-blende heterointerfaces in the InP nanowire.
\end{abstract}

DOI: 10.1103/PhysRevB.85.075324

PACS number(s): 78.67.Uh, 71.70.Ej, 78.55.-m, 78.67.Hc

\section{INTRODUCTION}

Semiconductor quantum dots (QDs) are called artificial atoms because of their atom-like discrete electronic structure due to quantum confinement. Exciton spins confined in a QD can relax via a wide variety of interaction and/or scattering; ${ }^{1-3}$ specific examples are valence band mixing, ${ }^{4-7}$ Coulomb interaction with environmental charge fluctuation, ${ }^{8-10}$ and nuclear spin fluctuation. ${ }^{11,12}$ QDs embedded in semiconductor nanowires (NWs) ${ }^{13,14}$ (hereafter called NW-QDs), i.e., combined zero-dimensional and one-dimensional structures, are among the most promising candidates for realizing singlephoton detectors ${ }^{15}$ and on-demand single-photon sources ${ }^{16}$ with high coherences and long dephasing times. Fast dephasing of carriers in NW structures fabricated by topdown techniques such as photo/electron-beam lithography and reactive ion/plasma etching is improved by the reduction of surface traps induced by defects and/or disorders within epitaxially grown bottom-up single or multi core(-shelled) NW structures. ${ }^{17}$ Emitter dephasing and polarization instability set up bit errors and debase the communication distance limit when implementing phase and polarization encoding quantum key distribution protocols. ${ }^{18}$ In this paper we investigate the transverse and longitudinal exciton-spin relaxation times. The transverse exciton-spin relaxation time of our NW-QD, caused by fluctuation in surrounding excess charges in the NW, is suppressed with decreasing excitation power, which was observed by first-order photon correlation measurements under nonresonant excitation condition. The longitudinal exciton-spin relaxation time was evaluated by the degree of random polarization in the time-integrated photoluminescence. The degree of the random polarization component is obtained by Mueller analysis of the experimental results of a full polarization measurement based on Stokes parameters.

\section{SAMPLE PREPARATION AND MEASUREMENT SETUP}

Arrays of InAsP QDs embedded in InP NWs were synthesized by selective area metal-organic vapor phase epitaxy. An $\mathrm{InP}$ (111)A wafer was covered with $30 \mathrm{~nm}$ of $\mathrm{SiO}_{2}$. By electron beam lithography and wet-etching, 40- to 60-nm-diameter openings were created to form NW nucleation sites (Fig. 1). At a growth rate of $3 \mathrm{~nm} / \mathrm{s}$, the first 1- $\mu \mathrm{m}$-long segment of InP was grown by adding trimethylindium and tertiarybutylphosphine (TBP) to the MOVPE reactor at $640{ }^{\circ} \mathrm{C}$. To form the QDs the temperature was lowered to $580{ }^{\circ} \mathrm{C}$ and arsine $\left(\mathrm{AsH}_{3}\right)$ was added to the reactor (V/III ratio 340; partial pressure, TBP: $\mathrm{AsH}_{3} 3: 1$ ). At a growth rate of $3 \mathrm{~nm} / \mathrm{s}$ an 8 - to $10-\mathrm{nm}$ layer of InAsP was created, forming the QDs. To cover the QDs with an InP shell, InP growth was first performed at $580{ }^{\circ} \mathrm{C}$. To finalize the NW-QDs, a second $1-\mu \mathrm{m}$ segment of InP was grown at $640{ }^{\circ} \mathrm{C}$.

Single NW-QD photoluminescence (PL) spectroscopy was performed by standard micro-PL measurements in the far field using a microscope objective as shown in Fig. 1. The sample was cooled to $5 \mathrm{~K}$ in a ${ }^{4} \mathrm{He}$ flow cryostat. The excitation laser beam traveling along the NW growth direction was focused on the sample surface with a microscope objective $(\mathrm{NA}=0.42)$. The NW-QD emission was collected by the same objective lens, dispersed with a double $(f=1.0 \mathrm{~m})$ grating spectrometer under zero magnetic field and a triple $(f=0.64 \mathrm{~m})$ grating spectrometer under a magnetic field and was detected with a liquid-nitrogen-cooled InGaAs photodiode array. The typical exposure time to obtain a spectrum with a high signal-to-noise ratio was $1 \mathrm{~s}$.

\section{BASIC OPTICAL PROPERTIES}

Figure 2(a) shows the excitation power dependence of the PL peak intensities of $X^{0}$ and $X X^{0}$. Excitation was carried out 


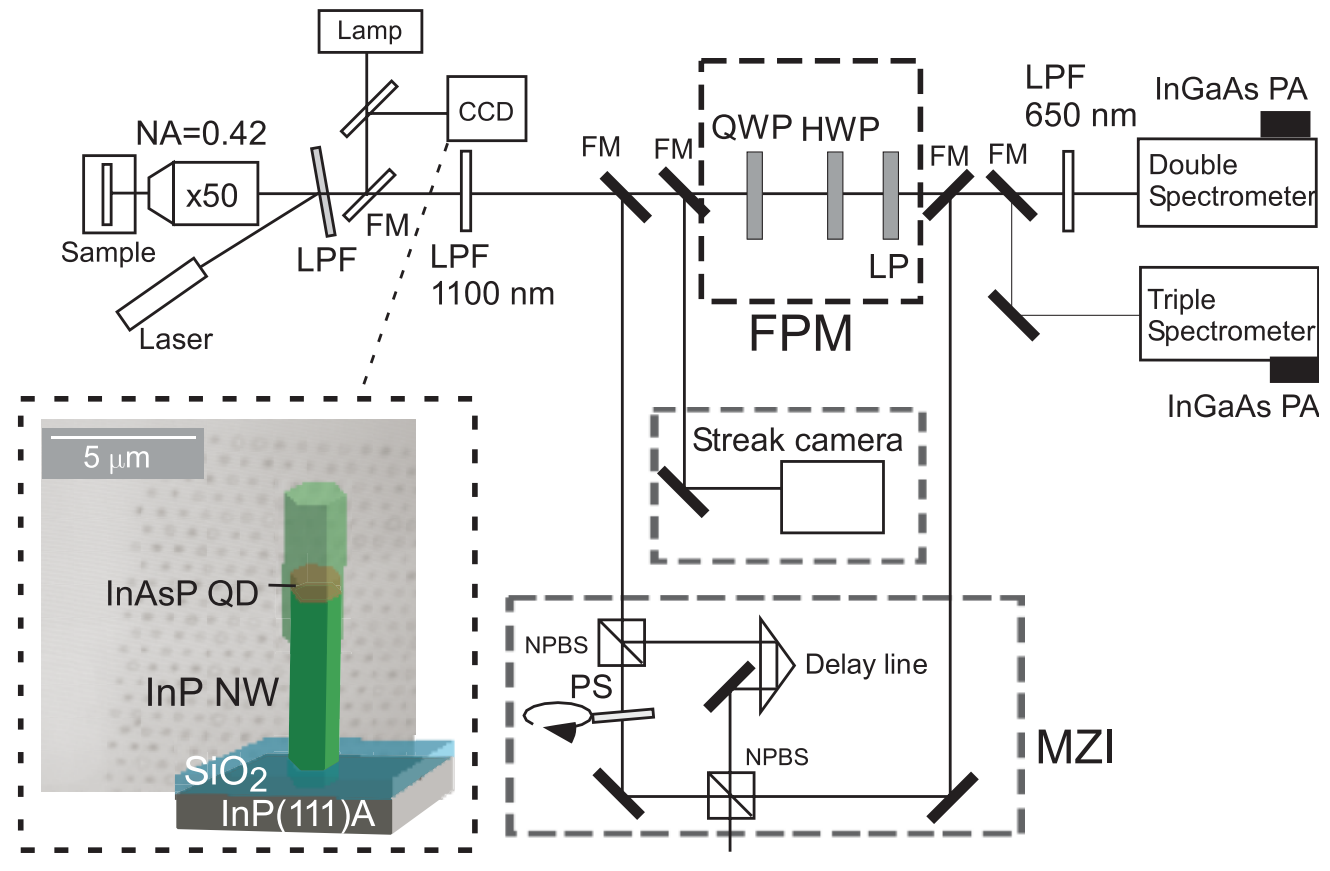

FIG. 1. (Color online) Schematic of the experimental setup. First-order photon correlation measurements were performed by measuring the PL passing through a Mach-Zehnder interferometer (MZI). The photon count was displayed as a function of the optical path delay. Full polarization measurements (FPMs) were performed using a set of quarter-wave plate (QWP), half-wave plate (HWP), and linear polarizer (LP). The PL decay time constant was measured using a streak camera. LPF, long-wave-pass filter; FM, flipper-mounted mirror; PS, phase shifter; NPBS, nonpolarizing beam splitter; PA, photodiode array. Inset: Microscope image of the sample surface and schematic of the NW-QD grown on InP (111)A substrate.

with a pulsed Ti:sapphire laser at a wavelength of $\sim 800 \mathrm{~nm}$ $(1.55 \mathrm{eV})$ and a repetition rate of $76 \mathrm{MHz}$. At a low excitation intensity, $X^{0}$ is centered at $\sim 1.023282 \mathrm{eV}$, with a $46-\mu \mathrm{eV}$ FWHM. With increasing excitation power, the PL intensity of $X^{0}$ increases linearly and saturates at an excitation power of $\sim 2 \mu \mathrm{W}$, while an additional line, labeled $X X^{0}$, appears, with its PL intensity increasing superlinearly. This indicates the origin of the PL peaks from neutral exciton and biexciton recombination. Figure 2(b) shows time-integrated PL spectra of a single NW-QD using a two-color excitation method. ${ }^{20} \mathrm{We}$ used a continuous-wave (cw) diode laser $(2.33 \mathrm{eV}, 532 \mathrm{~nm})$, which is above the bandgap energy of InP, and a cw Ti:sapphire laser $(1.35 \mathrm{eV}, 920 \mathrm{~nm})$ below the bandgap energy, for twocolor excitation. The excitation energy of the $1.35-\mathrm{eV}$ laser is still above the discrete levels in InAsP NW-QDs and creates electron-hole pairs in continuum states, ${ }^{21-24}$ it is estimated by PL excitation measurements (not shown). Although $X^{*}$ PL (charged exciton) is dominant under excitation condition (II; $2.33 \mathrm{eV}), X_{0}$ appears under two-color excitation condition (II) $+(\mathrm{I} ; 1.35 \mathrm{eV})$, which corresponds to the PL spectra under high-power excitation of (I). Note that the power of the $2.33-\mathrm{eV}$ laser (I) is sufficiently low so as not to allow observation of any PL emission, which suggests that the created carriers are almost entirely used for the screening of the internal electric field induced by the dipoles at the wurtzite and zinc-blende heterointerfaces $^{25}$ in the InP NW. Although InP NWs with a wurtzite crystal structure grown on InP (111)A substrate show type II optical transitions with lifetimes of the order of $100 \mathrm{~ns},{ }^{26,27}$ the decay time constant of the specific InP NWs presented in this paper is as short as $\sim 13 \mathrm{~ns}^{28}$ (not shown). This indicates that this type of $\mathrm{InP} \mathrm{NW}$ exhibits a mixed wurtzite and zinc-blende crystal structure. ${ }^{25}$ In Fig. 2(c), the Zeeman energy $\left(\Delta E_{\mathrm{ze}}\right)$ and diamagnetic shift $\left(\Delta E_{\text {diam }}\right)$ of a single NWQD are plotted against the external magnetic field along the growth direction (Faraday geometry). By fitting with $g_{\text {ex }} \mu_{\mathrm{B}} B_{z}$ and $\gamma_{2} B_{z}^{2},{ }^{29}$ where $\mu_{\mathrm{B}}$ is the Bohr magneton, we obtained an exciton $g$ factor of $\left|g_{\text {ex }}\right|=0.4$ and a diamagnetic coefficient of $\gamma_{2}=23.2 \pm 0.3 \mu \mathrm{eV} / \mathrm{T}^{2} \cdot \gamma_{2}$ is a good indicator of the degree of the lateral confinement potential. Compared to reports on small $\gamma_{2}$ values $(\ll 10 \mu \mathrm{eV})$ in type I InAs/GaAs QDs ${ }^{30,31}$ and InP/GaAs QDs, ${ }^{32,33}$ our observed $\gamma_{2}$ is larger than for typical type I QDs but smaller than for type II InP/GaAs QDs. ${ }^{34}$ The Bohr radius is deduced to be $\sim 7 \mathrm{~nm}$ using the effective masses in InP. The $X^{0}$ PL decays exponentially with an evaluated decay time constant $\tau_{r} \sim 2 \mathrm{~ns}$, obtained by the fitting [solid line in Fig. 2(d)], which suggests that the investigated InAsP QD exhibits a type I transition and thus a zinc-blende-like crystal structure. These observed results and the quantum-disk-like structure (height, $\sim 9 \mathrm{~nm}$; diameter, $\sim 100 \mathrm{~nm})^{19}$ indicate that the $X^{0}$ initial state is localized in a type I confinement potential as well as a relatively weak lateral confinement of the InAsP QD embedded in the InP NW. In what follows we focus on $X^{0}$, which generates the single-photon state estimated by the second-order photon correlation measurements presented in Ref. 19.

\section{TRANSVERSE EXCITON-SPIN RELAXATION}

The Mach-Zehnder interferometer inserted in the optical path was used to perform first-order photon correlation measurements on single NW-QD $X^{0}$ emission. This is a type of time-domain spectroscopy called single-photon Fourier 

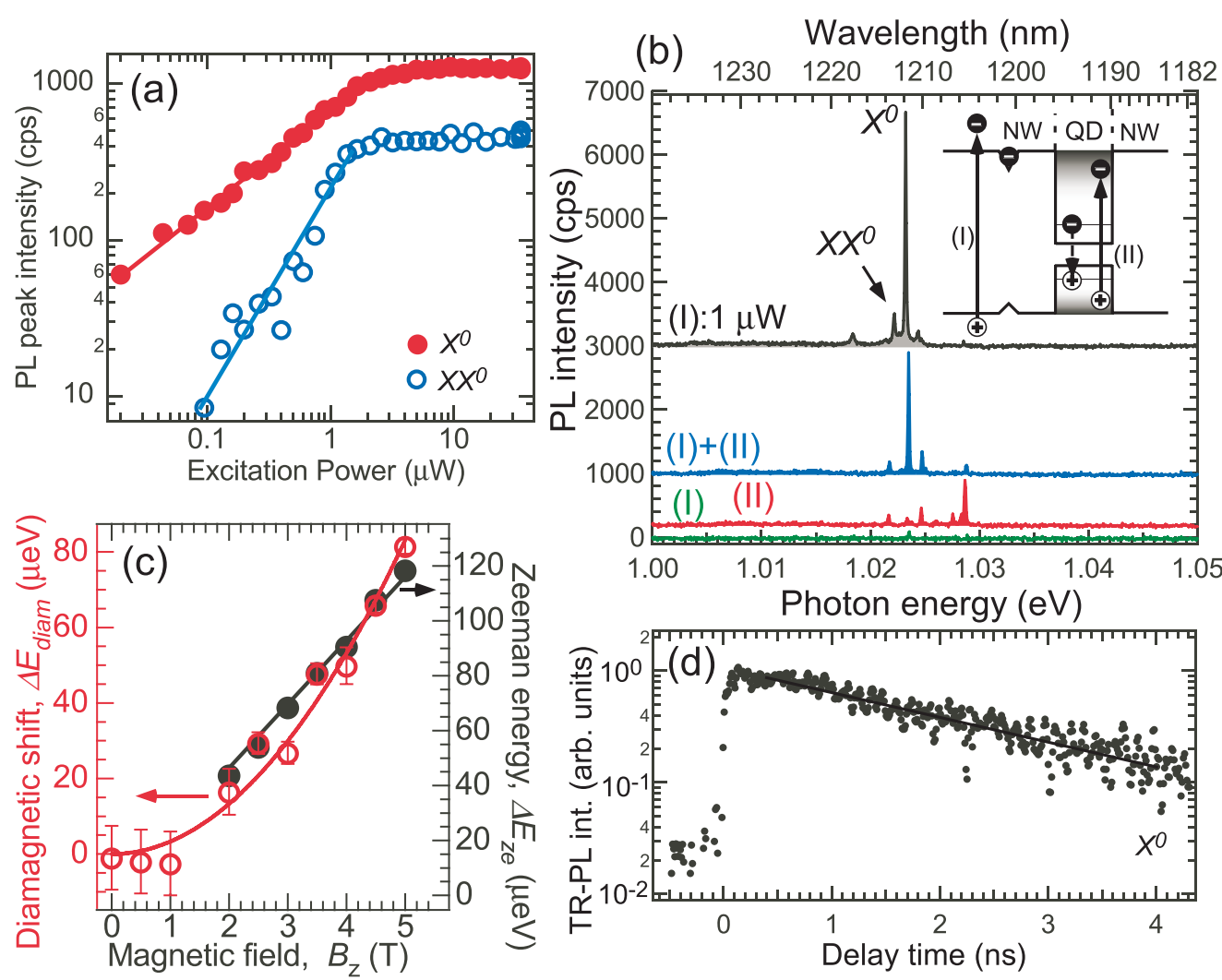

FIG. 2. (Color online) (a) PL intensities of $X^{0}$ and $X X^{0}$ peaks as a function of excitation power. Red and blue lines are guides for the eye. (b) PL spectra of an InAsP NW-QD obtained under different excitation conditions. $X^{0}$ PL appears under two-color excitation (I) + (II), which corresponds to the PL spectra under laser (I) high-power excitation at $1 \mu \mathrm{W}$. Excitation conditions are as follows: (I) a green 2.33-eV laser creates electrons and holes in an InP NW; (II) an NIR 1.35-eV laser creates excitons in an InAsP NW-QD. Laser powers are $10 \mathrm{nW}$ (I) and $25 \mu \mathrm{W}$ (II). Inset: Schematics energy band diagram. Graded areas in the InAsP QD schematic indicate continuum states. (c) Zeeman energy and diamagnetic shift of $X^{0}$. Filled (black) circles and open (red) circles represent experimental data, the (black) line and (red) curve are fits to the data with $\left|g_{\mathrm{ex}}\right| \mu_{\mathrm{B}} B_{z}$ and $\gamma_{2} B_{z}^{2}$, respectively. $\left|g_{\mathrm{ex}}\right|=0.4$ and $\gamma_{2}=23.2 \pm 0.3 \mu \mathrm{eV} / \mathrm{T}^{2}$. (d) Time-resolved PL of $X^{0}$ [filled (black) circles] under type (I; pulsed) excitation at $0.1 \mu \mathrm{W}$. The $X^{0}$ decay time of $\sim 2 \mathrm{~ns}$ is fitted with a single-exponential function [solid (black) line].

spectroscopy first demonstrated by Kammerer et al. ${ }^{35}$ The cw photoexcitation was carried out using a He-Ne laser for NW barrier excitation. Rotating a thin glass plate in one of the interferometer arms enables fine-tuning of the relative phase between the two arms.

Short-period fringe evolution of single-NW-QD $X^{0}$ exciton $\mathrm{PL}$ is shown in the inset in Fig. 3(a) at $\tau \sim 10$ ps. The fringe evolution as a function of delay $\tau$ is given by $I(\tau)=I_{0}[1+$ $\left.V(\tau) \cos \left(E_{0} \tau / \hbar+\theta\right)\right]$, where $E_{0}, I_{0}, V(\tau)$, and $\theta$ are the central detection energy $\left(\sim 1.023282 \mathrm{eV}\right.$ for $\left.X^{0}\right)$, the averaged signal intensity, the visibility contrast given by the modulus of the Fourier transform of the intensity spectrum, and the phase, respectively. By varying the time delay $\tau$, interference fringes of single-photon events are recorded. The visibility contrast $V(\tau)$ decays with increasing delay time $\tau$ between the two arms, and the coherence time is measured from the decay contrast of $V(\tau)$. The visibility curves shown in Fig. 3(a) decay following almost-single-exponential functions in the investigated range of excitation power, suggesting that the spectral diffusion phenomenon is in the unconventional motional narrowing regime, ${ }^{8-10} \Sigma \tau_{\mathrm{f}} / \hbar<1 . \Sigma=2 \Delta E_{s} \tau_{\mathrm{f}} / \sqrt{\tau_{\text {esc }} \tau_{\text {cap }}}$ is the modulation amplitude caused by an individual point charge and $\Delta E_{s}$ is the energy saturation value. ${ }^{9}$ The rate of correlation between the confined exciton and the point charges in the NW is expressed by the combination of capture and escape rates, $1 / \tau_{\mathrm{f}}=1 / \tau_{\text {cap }}+1 / \tau_{\text {esc }}$. In this regime the observed transverse exciton-spin relaxation time $T_{2}$ is given by $1 / T_{2}=(\Sigma / \hbar)^{2} \tau_{\mathrm{f}}$ and the line shape remains Lorentzian under Gaussian fluctuations.

With decreasing excitation power, the measured $T_{2}$ increases and saturates at $\sim 45$ ps as shown in Fig. 3(b). This indicates that the environmental charge fluctuations were sufficiently suppressed under weak excitation and that the observed $T_{2}$ is limited by the relaxation processes in the QD. ${ }^{1,2}$ The observed low-power (lp) saturation value of $T_{2}^{(\mathrm{lp})} \sim 45 \mathrm{ps}$ is about 10 times shorter than that measured under resonant excitation using various different experimental methods and materials. $^{36,37}$ With increasing excitation power, $T_{2}$ decreases gradually and saturates to $T_{2}^{(\mathrm{hp})} \sim 18 \mathrm{ps}$ above the excitation power of $\sim 0.4 \mu \mathrm{W}$. The prolonged transverse exciton-spin relaxation time corresponds to the red-shift, $\Delta E$ of $X^{0}$, as shown in Fig. 3(b) (right axis). This energy shift is attributed to the Stark effect due to the internal electric field induced by dipoles at the wurtzite and zinc-blende heterointerfaces in the $\mathrm{NW}^{25}$ and to the screening of the internal field by the photoexcited carriers in the InP NW barrier. 

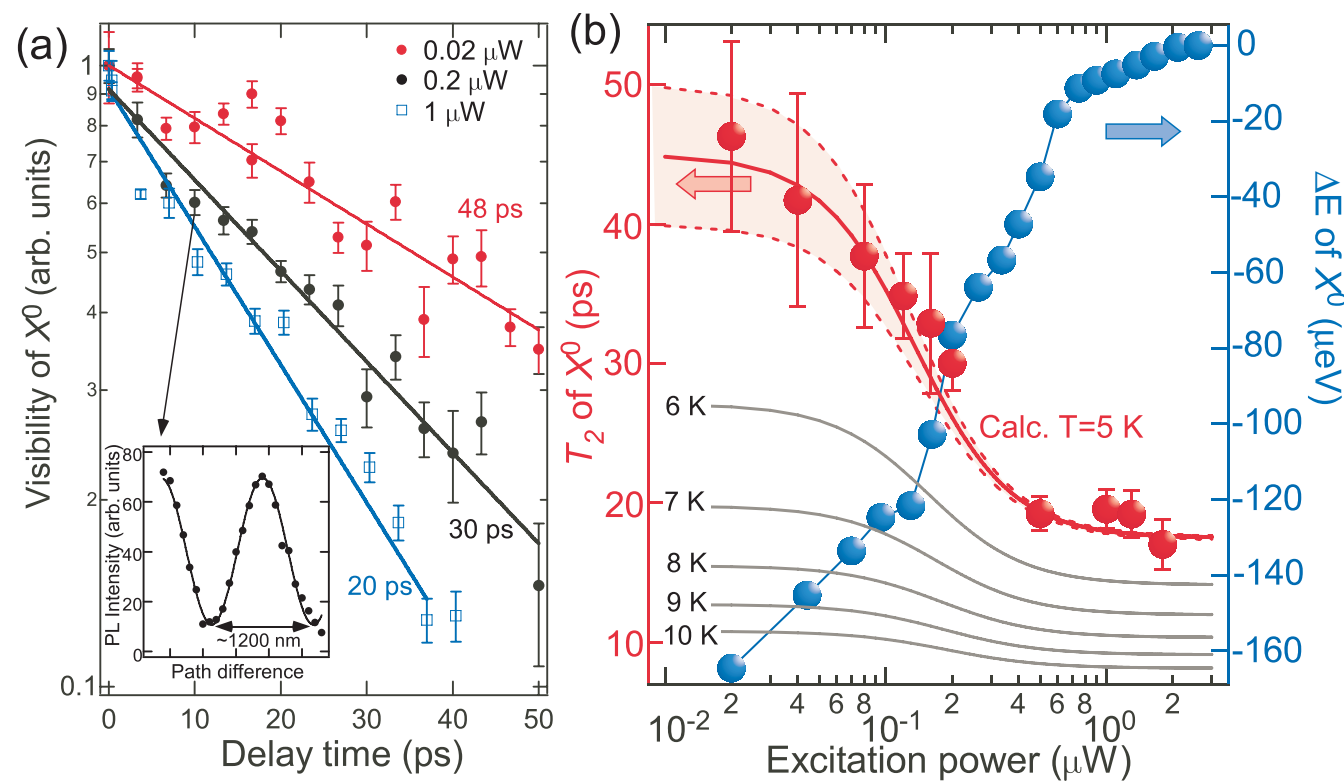

FIG. 3. (Color online) (a) Visibility plot for single-dot $X^{0}$ PL. Inset: Short-period fringe evolution of PL at a delay time of 10 ns. (b) Excitation power $(P)$ dependence of $T_{2}$ [filled (red) circles] under He-Ne laser excitation, with the corresponding solid (red) curve representing the calculation results using Eq. (1), and the PL peak energy of $X^{0}, \Delta E=E(P)-E(2 \mu \mathrm{W})$ [filled (blue) circles]. The area between the dashed (red) curves indicates the calculation results of $T_{2}$ for $T_{2}^{(\mathrm{lp})}=45 \pm 5 \mathrm{ps}$. The $P$ dependence of $T_{2}$ is calculated by the motional narrowing fluctuation for $T=6-10 \mathrm{~K}$ [solid (gray) curves] under fixed phonon energies, $E_{1} \sim 1 \mathrm{meV}$ and $E_{2}=42.4 \mathrm{meV}$.

For simplicity, we adopt the approach of Favero et al., ${ }^{9}$ where the acoustic and optical phonon-assisted capture and escape rates are expressed as

$$
\begin{aligned}
& \frac{1}{\tau_{\text {cap }}}=\frac{1}{\tau_{1}}\left[1+n_{1}(T)\right]+\frac{1}{\tau_{2}}\left[1+n_{2}(T)\right], \\
& \frac{1}{\tau_{\text {esc }}}=\frac{1}{\tau_{1}} n_{1}(T)+\frac{1}{\tau_{2}} n_{2}(T)+\frac{1}{\tau_{3}} \frac{P^{2}}{P^{2}+\left(\sqrt{3} P_{0}\right)^{2}} .
\end{aligned}
$$

$n_{1(2)}, \tau_{1(2)}, \tau_{3}, P$, and $P_{0}$ are the Bose-Einstein occupation factors of acoustic (optical) phonons given by $1 /\left[\exp \left(E_{1(2)} / k_{\mathrm{B}} T\right)-1\right]$, the correlation times between point charge in the InP NW and acoustic (optical) phonon, the correlation time of Auger processes, the excitation power, and the characteristic excitation power of the appearance of Auger processes, which gives the inflection point of the calculated $T_{2}$ value, respectively. $E_{1} \sim 1 \mathrm{meV}^{8-10}$ and $E_{2}=42.4 \mathrm{meV}$ are the acoustic phonon mean energy and the optical phonon energy of bulk InP, which indicates that optical phonon-assisted escape processes are negligibly small at low temperatures and which allows for the following simplification: $n_{2}(5 \mathrm{~K}) \approx 0$. Substituting both excitation power limits, i.e., $P \gg P_{0}$ and $P \ll P_{0}$, into Eq. (1), we can derive empirical relations among the three correlation times, $\tau_{2}=(0.875 \pm 0.05) \times$ $\mathrm{ps}^{1 / 2} \tau_{1}^{1 / 2}$ and $\tau_{3}=(6.6 \pm 1.0) \times \tau_{1}$ [see Eqs. (A1) and (A2) in the Appendix]. These relations suggest that the optical phonon-assisted capture process and acoustic phonon-assisted escape process are dominant under the present experimental conditions. Both trends are attributed to the strong coupling between optical phonon and point charges and to the low activation energy of point charges. We can thus simulate the approximated excitation power dependence of $T_{2}$ by using the above relations, together with the empirical parameter deduced from experimental results, $\Delta E_{s}=180 \mu \mathrm{eV}$, as shown in Fig. 3(b), and one fitting parameter, $P_{0}=0.12 \mu \mathrm{W}$. As displayed in Fig. 3(b), we observe good agreements between the experimental $T_{2}$ values and the calculations [solid (red) curve] using a correlation time $\tau_{1}=250 \mathrm{ps}$, which is a value similar to those reported in Refs. 9 and 10.

\section{LONGITUDINAL EXCITON-SPIN RELAXATION}

In order to investigate the longitudinal exciton-spin relaxation time of a single NW-QD, a quarter-wave plate (QWP), half-wave plate (HWP), and linear polarizer (LP) were placed in front of the spectrometer. The transmission axis of the LP was set horizontal in the laboratory frame. $\theta$ and $\rho$ are the relative angles of the QWP's and HWP's fast axes with respect to the transmission axis of the LP. A He-Ne laser was used as a quasirandomly polarized excitation source and was focused on the sample surface by the microscope objective. Figure 4(a) shows a two-dimensional (2D) plot of normalized PL intensities $I_{\mathrm{PL}}(\theta, \rho) /\left\{2\left\langle I_{\mathrm{PL}}^{\theta}(\rho)\right\rangle\right\}$ as a function of $\rho$ and $\theta$. This checkered-flag-like pattern signifies that the observed PL is elliptically polarized. There are theoretical and experimental reports that the elliptical polarization of photons originating from excitons of a single QD can be well explained by the anisotropic heavy-hole/light-hole mixing induced by the inhomogeneous piezoelectric field distribution. $4,6,7,38,39$ Although the amplitude of the oscillation reaches a maximum at $\theta \approx 59^{\circ}$, which is the cancellation condition of circular polarization components by the QWP, the observed amplitude is $<1$ [Fig. 4(c)], suggesting that the observed photon state includes random polarization components.

To analyze the obtained results for $X^{0}$, we introduce normalized Stokes parameters ${ }^{40,41}$ with a random polarization 

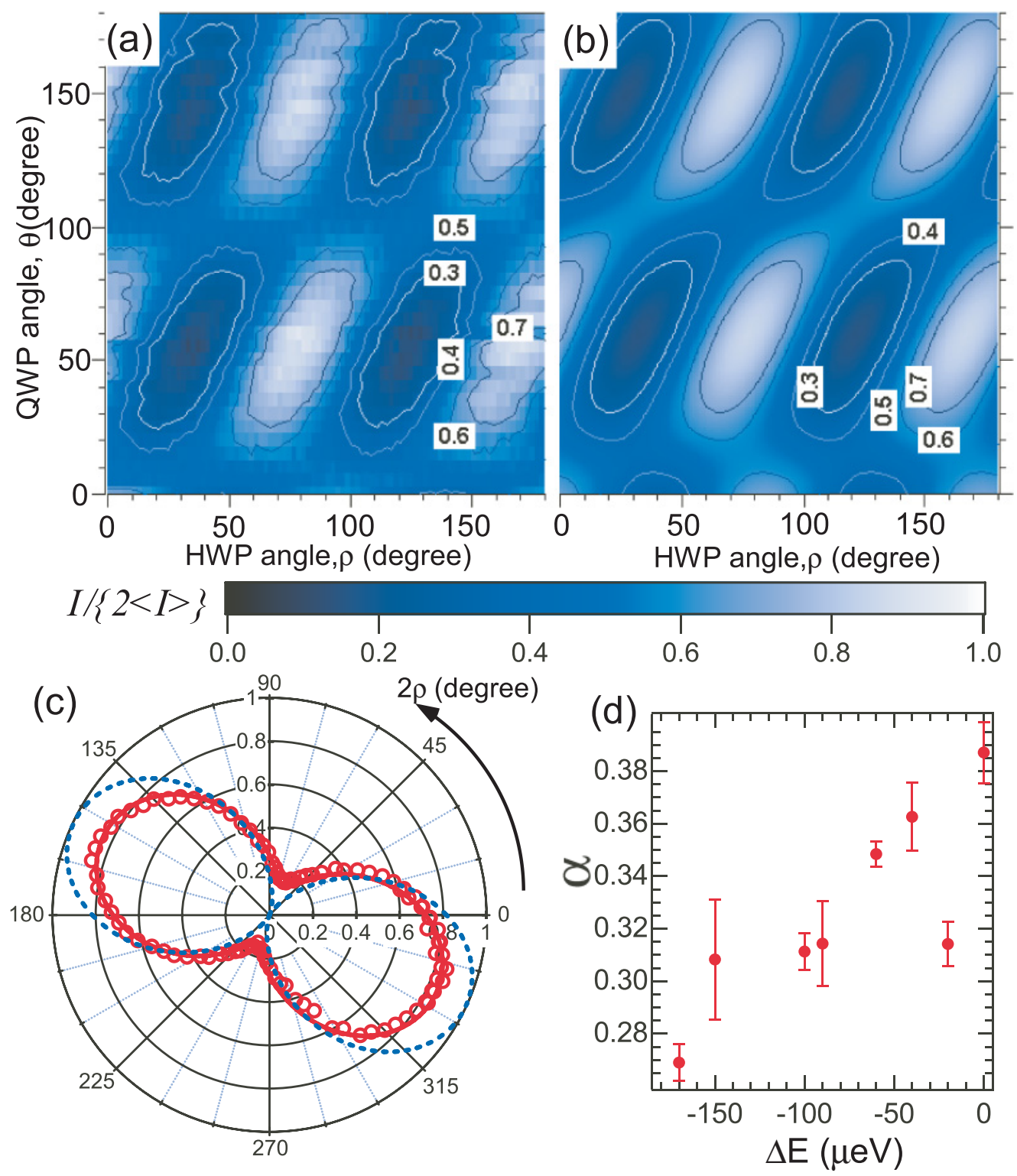

FIG. 4. (Color online) (a) 2D plot of normalized PL intensities of $X^{0}$ as a function of $\rho$ and $\theta$. (b) Mueller calculus' results by using the components $\{1,0.32,-0.6,0.14\}$. (c) Polar plot of the PL intensity of $X^{0}$. The detection angle $(2 \rho)$ dependence of $I_{\mathrm{PL}}\left(59^{\circ}, \rho\right) /\left\{2\left\langle I_{\mathrm{PL}}^{59^{\circ}}(\rho)\right\rangle\right\}$ (open (red) circles). Solid (red) and dashed (blue) lines are Mueller calculus' results for $\alpha=0.3,\{1,0.32,-0.6,0.14\}$ and $\alpha=0$, $\{1,0.32 / 0.7,-0.6 / 0.7,0.14 / 0.7\}$, with the latter being perfectly coherent light with a fixed ratio of the three polarization components. All angles are measured in the same laboratory frame. (d) $\Delta E$-dependence of the DRP $\alpha$ under fixed ratios of Stokes parameters of the polarization components $\left\{S_{1}, S_{2}, S_{3}\right\}$.

component, $\left\{1, S_{1}(\alpha), S_{2}(\alpha), S_{3}(\alpha)\right\}$, where $S_{i}(\alpha)=(1-\alpha) S_{i}$, $\alpha$ is the degree of random polarization (DRP), $\alpha\{1,0,0,0\}$, and $S_{i}$ are the Stokes parameters of the coherent components. Applying the traditional $4 \times 4$ Mueller matrices, the polarized light propagation can be simulated. Here we assume that three polarization optics are ideal and common in the Mueller calculus. The intensity passing through three polarization optics is expressed as

$$
I=\frac{1}{2}\left[1+(1-\alpha) \sqrt{S_{3}^{2}+b(\theta)^{2}} \cos (4 \rho-2 \delta(\theta))\right],
$$

where $b(\theta)=S_{1} \cos (2 \theta)+S_{2} \sin (2 \theta)$ and $\delta(\theta)=0.5 \arctan$ $\left(S_{2}^{\prime} / S_{1}^{\prime}\right)$, which is the tilting angle from the horizontal axis of the linear polarization components after the light has passed through the QWP $(\theta)$ : QWP $(\theta)\left\{1, S_{1}, S_{2}, S_{3}\right\} \rightarrow\left\{1, S_{1}^{\prime}, S_{2}^{\prime}, S_{3}^{\prime}\right\}$.

Under the cancellation condition of circular polarization components by the QWP, $\theta=0.5 \arctan \left(S_{2} / S_{1}\right)$, the amplitude of oscillation reaches the maximum. Substituting $\theta=0.5 \arctan \left(S_{2} / S_{1}\right)$ and $\alpha=0$ into Eq. (2), the amplitude becomes exactly 1 . Note that this is suitable only for the limiting case where the exciton-spin-flip time, i.e., the longitudinal exciton-spin relaxation time $T_{1}$, is much longer than the decay time $\tau_{r}$. Figure 4(c) shows the polar plot at $\theta=59^{\circ}$ of the observed $I_{\mathrm{PL}}\left(59^{\circ}, \rho\right) /\left\{2\left\langle I_{\mathrm{PL}}^{59^{\circ}}(\rho)\right\rangle\right\}$. The maximum amplitude, $0.85=1-\alpha / 2$, is $<1$. By using $\alpha=0.3$ and $\theta=0.5 \arctan \left(S_{2} / S_{1}\right)=59^{\circ}$, we can deduce 
the Stokes parameters. Assuming the Stokes parameters $\{1,0.32,-0.6,0.14\}$, we can reproduce not only the amplitude of $I_{\mathrm{PL}}(\theta, \rho) /\left\{2\left\langle I_{\mathrm{PL}}^{\theta}(\rho)\right\rangle\right\}$, but also its pattern, very well, as shown in Figs. 4(b) and 4(c). The observed DRP is evaluated as $0.3\left[=1-\sqrt{S_{1}(\alpha)^{2}+S_{2}(\alpha)^{2}+S_{3}(\alpha)^{2}}\right]$, suggesting that $T_{1} \sim 0.7 \times \tau_{r}=1.4 \mathrm{~ns}$ [Fig. 2(d)]. Note that the random polarization component can be observed in the time-integrated PL as a case of $T_{1} \leqslant \tau_{r}$. The ratio between $T_{1}$ and $\tau_{r}$ is expressed as $T_{1} / \tau_{r}=\int d t e^{-t / T_{1}} / \int d t e^{-t / \tau_{r}}=1-\alpha$ within the relaxation time approximation. Figure 4(d) shows the $\Delta E$ dependence of $\alpha$ under the assumption of a fixed ratio of the three polarization components. $\alpha$ decays slightly with decreasing $\Delta E$, implying that the overlap integral of electron and hole envelope wave functions, $\left\langle\varphi_{e} \mid \varphi_{h}\right\rangle$, decreases due to the internal field. ${ }^{25}$ In general, the exciton spin in a quantum well at low temperatures can mainly flip via short- and longrange exchange interactions, i.e., via the Maialle-AndradaSham mechanism. ${ }^{3}$ In the motional narrowing type, $T_{1}$ is given by the fluctuation of an effective magnetic field with an angular precession frequency, $\Omega$, originating from exchange interaction, $\Delta$, between electron and hole and the scattering time, $\tau_{\mathrm{c}}$, of center-of-mass motion of the exciton, $1 / T_{1} \approx\left\langle\Omega^{2}\right\rangle \times \tau_{\mathrm{c}} \approx$ $(\Delta / \hbar)^{2} \times \tau_{\mathrm{c}} \cdot{ }^{1,2} \Delta$ and $1 / \tau_{r}$ are approximately proportional to $\left|\left\langle\varphi_{e} \mid \varphi_{h}\right\rangle\right|^{2}$, indicating $\alpha \propto 1-\left|\left\langle\varphi_{e} \mid \varphi_{h}\right\rangle\right|^{-2}$. Both observed results, the $\Delta E$ dependence of $\alpha$ and the high degree of linear polarization, $0.98\left(=\sqrt{S_{1}^{2}+S_{2}^{2}}\right)$, suggest that the observed $T_{1}$ time is dominated by short-range exchange interactions corresponding to strong light-hole/heavy-hole mixing.

\section{SUMMARY}

We investigated the optical properties of a single QD embedded in a vertically standing NW structure and measured the transverse and longitudinal exciton-spin relaxation times. The transverse exciton-spin relaxation is induced by fluctuations of environmental excess charges in the NW and can be reduced by decreasing the excitation intensity. The experimentally obtained transverse exciton-spin relaxation times, which were evaluated as $T_{2}=45-18 \mathrm{ps}$, can be reproduced by the unconventional motional narrowing theory. To determine the longitudinal exciton-spin relaxation time, we performed full polarization measurements and Mueller calculus. The longitudinal exciton-spin relaxation times, $T_{1}=1.4 \mathrm{~ns}$, evaluated by the random polarization components are shorter than the decay time. A decreasing random polarization component of emitted photons from exciton states with decreasing emission energy was observed due to the reduction of the overlap integral of electron and hole envelope wave functions. We believe that the obtained results can contribute to the understanding of effective exciton-spin relaxation mechanisms in nanostructures.

\section{ACKNOWLEDGMENTS}

This work was supported in part by Grant-in-Aid for Young Scientists (B) No. 20760002 and GCOE-GSIST, Hokkaido University. M.K., N.A., and V.Z. acknowledge funding from NWO; C.H. acknowledges financial support in the form of a JSPS Fellowship for Foreign Researchers. H.S. acknowledges Professor S. Muto, Professor S. Adachi and Dr. R. Kaji for fruitful discussions.

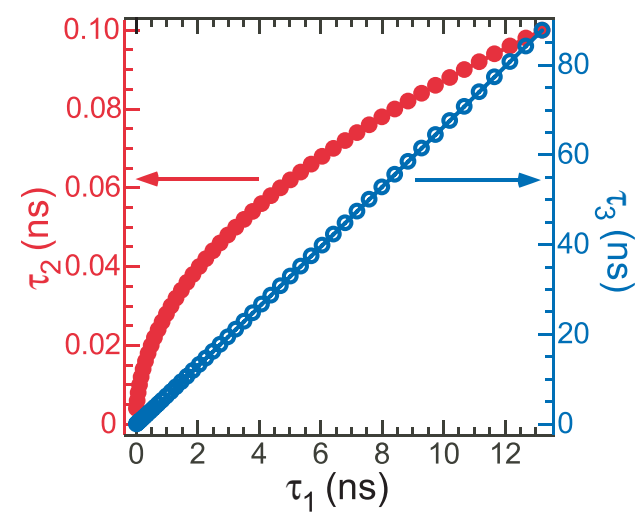

FIG. 5. (Color online) Calculation results for $\tau_{2}$ vs. $\tau_{1}$ [filled (red) circles] and $\tau_{3}$ vs. $\tau_{1}$ [open (blue) circles]. The solid (red) curve is a fit using a square root function. The solid (blue) line is a fit using a linear function.

\section{APPENDIX: DERIVATION OF THE EMPIRICAL RELATION AMONG THREE CORRELATION TIMES}

In the low- and high-power limits, the escape rate in Eq. (1) can be transformed to

$$
\frac{1}{\tau_{\mathrm{esc}}} \approx \begin{cases}0.1 / \tau_{1}, & P \ll P_{0}, \\ 0.1 / \tau_{1}+1 / \tau_{3}, & P \gg P_{0},\end{cases}
$$

where the Bose-Einstein occupation factors of acoustic and optical phonons are $n_{1}(5 \mathrm{~K}) \approx 0.1$ and $n_{2}(5 \mathrm{~K}) \approx 0$. The capture rate in Eq. (1) can be described as $1 / \tau_{\text {cap }} \approx 1.1 / \tau_{1}+$ $1 / \tau_{2}$. In the low-power limit, $P \ll P_{0}$, the saturation value of the transverse exciton-spin relaxation rate is given by

$$
\frac{1}{T_{2}^{(\mathrm{lp})}} \approx\left(\frac{2 \Delta E_{s}}{\hbar}\right)^{2} \frac{1.1 \tau_{2}+\tau_{1}}{\left(1.2 \tau_{2}+\tau_{1}\right)^{3}} \frac{\tau_{1} \tau_{2}^{2}}{10}=\frac{1}{45 \mathrm{ps}},
$$

where the values of $T_{2}^{(\mathrm{lp})} \approx 45 \mathrm{ps}$ and $\Delta E_{s}=180 \mu \mathrm{eV}$ are deduced from Fig. 3(b). The calculation results are fitted with a square root function,

$$
\tau_{2}=(0.87 \pm 0.05) \mathrm{ps}^{1 / 2} \times \tau_{1}^{1 / 2},
$$

as shown in Fig. 5.

In the high-power limit, $P \gg P_{0}$, by substituting $\tau_{2}=$ $0.87 \mathrm{ps}^{1 / 2} \times \tau_{1}^{1 / 2}$ into the capture and escape rates of Eq. (1), we obtain

$$
\begin{aligned}
\frac{1}{T_{2}^{(\mathrm{hp})} \approx} & \left(\frac{2 \Delta E_{s}}{\hbar}\right)^{2} \\
& \times \frac{a_{1} \tau_{1}^{2} \tau_{3}^{2}\left(a_{2} \tau_{1}^{3 / 2}+\tau_{1}^{2}+a_{3} \tau_{1}^{1 / 2} \tau_{3}+0.1 \tau_{1} \tau_{3}\right)}{\left(0.87 \mathrm{ps}^{1 / 2} \tau_{1}^{3 / 2}+a_{4} \tau_{1}^{1 / 2} \tau_{3}+\tau_{1} \tau_{3}\right)^{3}} \\
= & \frac{1}{18 \mathrm{ps}}, \quad a_{1}=0.7569 \mathrm{ps}, \quad a_{2}=0.957 \mathrm{ps}^{1 / 2}, \\
a_{3}= & 0.0957 \mathrm{ps}^{1 / 2}, \quad a_{4}=1.044 \mathrm{ps}^{1 / 2} .
\end{aligned}
$$

The value of $T_{2}^{(\mathrm{hp})} \approx 18 \mathrm{ps}$ is deduced from Fig. 3(b). The calculation results are fitted with a linear function,

$$
\tau_{3}=(6.6 \pm 1.0) \times \tau_{1},
$$

as shown in Fig. 5. 
*hirotaka@eng.hokudai.ac.jp

${ }^{1}$ F. Meier and B. P. Zakharchenya (eds.), Optical Orientation, Modern Problems in Condensed Matter Sciences (North-Holland, New York, 1984), Vol. 8, Chap. 3.

${ }^{2}$ E. L. Ivchenko and G. E. Pikus (eds.), Superlattices and Other Heterostructures, Springer Series in Solid-State Sciences (SpringerVerlag, Berlin, 1997), Vol. 110.

${ }^{3}$ M. Z. Maialle, E. A. de Andrada e Silva, and L. J. Sham, Phys. Rev. B 47, 15776 (1993).

${ }^{4}$ Y. Léger, L. Besombes, L. Maingault, and H. Mariette, Phys. Rev. B 76, 045331 (2007).

${ }^{5}$ K. Roszak, V. M. Axt, T. Kuhn, and P. Machnikowski, Phys. Rev. B 76, 195324 (2007).

${ }^{6}$ T. Beihadj, T. Amand, A. Kunold, C.-M. Simon, T. Kuroda, M. Abbarchi, T. Mana, K. Sakoda, S. Kunz, X. Marie, and B. Urbaszek, Appl. Phys. Lett. 97, 051111 (2010).

${ }^{7}$ S. Ohno, S. Adachi, R. Kaji, S. Muto, and H. Sasakura, Appl. Phys. Lett. 98, 161912 (2011).

${ }^{8}$ A. Berthelot, I. Favero, G. Cassabois, C. Voisin, C. Delalande, Ph. Roussignol, R. Ferreira, and J. M. Gérard, Nat. Phys. 2, 759 (2006).

${ }^{9}$ I. Favero, A. Berthlot, G. Cassabois, C. Voisin, C. Delalande, Ph. Roussignol, R. Ferreira, and J. M. Gérard, Phys. Rev. B 75, 073308 (2007)

${ }^{10}$ R. B. Patel, A. J. Bennett, K. Cooper, P. Atkinson, C. A. Nicoll, D. A. Ritchie, and A. J. Shields, Phys. Rev. Lett. 100, 207405 (2008).

${ }^{11}$ I. A. Merkulov, A. L. Efros, and M. Rosen, Phys. Rev. B 65, 205309 (2002).

${ }^{12}$ X. Xu, W. Yao, B. Sun, D. G. Steel, A. S. Bracker, D. Gammon, and L. J. Sham, Nature (London) 459, 1105 (2009).

${ }^{13}$ L. J. Lauhon, M. S. Gudiksen, D. Wang, and C. M. Lieber, Nature 420, 57 (2002).

${ }^{14}$ X. Duan, Y. Huang, Y. Cui, J. Wang, and C. M. Lieber, Nature 409, 66 (2001).

${ }^{15}$ M. P. van Kouwen, M. H. M. van Weert, M. E. Reimer, N. Akopian, U. Perinetti, R. E. Algra, E. P. A. M. Bakkers, L. P. Kouwenhoven, and V. Zwiller, Appl. Phys. Lett. 97, 113108 (2010).

${ }^{16}$ M. E. Reimer, M. P. van Kouwen, A. W. Hidma, M. H. M. van Weert, E. P. A. M. Bakkers, L. P. Kouwenhoven, and V. Zwiller, Nano Lett. 11, 645 (2011).

${ }^{17}$ P. Mohan, J. Motohisa, and T. Fukui, Nanotechnology 16, 2903 (2005).

${ }^{18}$ D. D. Awschalom, N. Samarth, and D. Loss, Semiconductor Spintronics and Quantum Computation (Springer, Berlin, 2002).

${ }^{19}$ S. N. Dorenbos, H. Sasakura, M. P. van Kouwen, N. Akopian, S. Adachi, N. Namekata, M. Jo, J. Motohisa, Y. Kobayashi, K. Tomioka, T. Fukui, S. Inoue, H. Kuman, C. M. Natarajan, R. H. Hadfield, T. Zijlstra, T. M. Klapwijk, V. Zwiller, and I. Suemune, Appl. Phys. Lett. 97, 171106 (2010).
${ }^{20}$ H. Gotoh, H. Sanada, H. Kamada, H. Yamaguchi, and T. Sogawa, Appl. Phys. Lett. 98, 032101 (2011).

${ }^{21}$ Y. Toda, O. Moriwaki, M. Nishioka, and Y. Arakawa, Phys. Rev. Lett. 82, 4114 (1999).

${ }^{22}$ J. J. Finley, A. D. Ashmore, A. Lemaître, D. J. Mowbray, M. S. Sckolnick, I. E. Itskevich, P. A. Maksym, M. Hopkinson, and T. F. Krauss, Phys. Rev. B 63, 073307 (2001).

${ }^{23}$ C. Kammerer, G. Cassabois, C. Voisin, C. Delalande, Ph. Roussignol, and J. M. Gérard, Phys. Rev. Lett. 87, 207401 (2001).

${ }^{24}$ A. Vasanelli, R. Ferreira, and G. Bastard, Phys. Rev. Lett. 89, 216804 (2002).

${ }^{25}$ B. Pal, K. Goto, M. Ilezawa, Y. Masumoto, P. Mohan, J. Motohisa, and T. Fukui, Appl. Phys. Lett. 93, 073105 (2008).

${ }^{26}$ A. Mishra, L. V. Titova, T. B. Hoang, H. E. Jackson, L. M. Smith, J. M. Yarrison-Rice, Y. Kim, H. J. Joyce, Q. Gao, H. H. Tan, and C. Jagadish, Appl. Phys. Lett. 91, 263104 (2007).

${ }^{27}$ Y. Masumoto, Y. Hirata, P. Mohan, J. Motohisa, and T. Fukui, Appl. Phys. Lett. 98, 211902 (2011).

${ }^{28}$ H. Sasakura, H. Kumano, I. Suemune, J. Motohisa, Y. Kobayashi, M. van Kouwen, K. Tomioka, T. Fukui, N. Akopian, and V. Zwiller, J. Phys.: Conf. Series 193, 012132 (2009).

${ }^{29}$ S. N. Walck and T. L. Reinecke, Phys. Rev. B 57, 9088 (1998).

${ }^{30}$ T. Nakaoka, T. Saito, J. Tatebayashi, and Y. Arakawa, Phys. Rev. B 70, 235337 (2004).

${ }^{31}$ C. Schulhauser, D. Haft, R. J. Warburton, K. Karrai, A. O. Govorov, A. V. Kalameitsev, A. Chaplik, W. Schoenfeld, J. M. Garcia, and P. M. Petroff, Phys. Rev. B 66, 193303 (2002).

${ }^{32}$ M. Hayne, R. Provoost, M. K. Zundel, Y. M. Manz, K. Eberl, and V. V. Moshchalkov, Phys. Rev. B 62, 10324 (2000).

${ }^{33}$ M. Sugisaki, H.-W. Ren, S. V. Nair, K. Nishi, and Y. Masumoto, Phys. Rev. B 66, 235309 (2002).

${ }^{34}$ B. Bansal, S. Godefroo, M. Hayne, G. Medeiros-Ribeiro, and V. V. Moshchalkov, Phys. Rev. B 80, 205317 (2009).

${ }^{35}$ C. Kammerer, G. Cassabois, C. Voisin, M. Perrin, C. Delalande, Ph. Roussignol, and J. M. Gérard, Appl. Phys. Lett. 81, 2737 (2002).

${ }^{36}$ D. Birkedal, K. Leosson, and J. M. Hvam, Phys. Rev. Lett. 87, 227401 (2001)

${ }^{37}$ K. Kuroda, T. Kuroda, K. Watanabe, T. Mano, K. Sakoda, G. Kido, and N. Koguchi, Appl. Phys. Lett. 90, 051909 (2007).

${ }^{38}$ E. Poem, J. Shemesh, I. Marderfeld, D. Galushko, N. Akopian, D. Gershoni, B. D. Gerardot, A. Badolato, and P. M. Petroff, Phys. Rev. B 76, 235304 (2007).

${ }^{39}$ M. Gong, W. Zhang, G-C. Guo, and L. He, Phys. Rev. Lett. 106, 227401 (2011).

${ }^{40}$ W. A. Shurcliff, Polarized Light (Harvard University Press, Cambridge, MA, 1962).

${ }^{41}$ E. Hecht, Am. J. Phys. 38, 1156 (1970). 